\title{
The evaluation of lightning return stroke current using measured fields
}

\begin{abstract}
In this paper an inverse procedure algorithm in the time domain is proposed to evaluate the wave shape of the lightning return stroke current along a lightning channel using measured electromagnetic fields obtained at different distances with respect to the lightning channel. Moreover, the proposed method was applied on an actual sample of lightning current and the results discussed accordingly. The proposed method takes into consideration all the field components which cover the full shape of the current. Thus the method can be helpful for preparing a lightning current data bank and setting a proper protection level for a power system based on local information.
\end{abstract}

Keyword: Lightning; Electromagnetic fields; Return stroke current 